Die Tatsache, dass in westlichen Industriestaaten - und eben auch in der Bundesrepublik Deutschland - autochthone Minderheiten nicht behinderter Erwachsener leben, die den in einer Gesellschaft üblichen Mindestanforderungen an die Schriftsprache nicht genügen, ist gemeinhin wenig bekannt und wird häufig mit Skepsis zur Kenntnis genommen. Selbst wenn in jüngerer Zeit, nicht zuletzt befördert durch die Ergebnisse der PISA-Studie, die prekäre Situation so genannter „funktionaler Analphabeten“ vermehrt in den Fokus von Öffentlichkeit und Politik kommt, so gibt es doch hinsichtlich sozialwissenschaftlicher Forschung und Diskurs immer noch eine große Lücke.Infolge struktureller Veränderungen der deutschen Wirtschaft, die verstärkt zu einem Verdrängungswettbewerb auf dem Arbeitsmarkt führten, wurde Ende der 1970er Jahre das Problem des funktionalen Analphabetismus sichtbar. Zunehmende Automatisierung und Rationalisierungsmaßnahmen

\title{
Erwachsenen- alphabetisierung
} bedingten den Wegfall von Arbeitsplätzen, auf denen bislang schriftsprachlich gering qualifizierte Arbeiter eine Beschäftigung fanden. Als Reaktion auf das Gewahrwerden eines solch erschreckend hohen Anteils von Menschen, die nicht oder nur sehr wenig lesen und schreiben können, setzte eine sozial- und bildungspolitisch motivierte Bewegung ein. Aus der anfänglich eher karitativ oder humanistisch motivierten Arbeit mit den Betroffenen erwuchs die Erkenntnis, Erwachsenenalphabetisierung als gesellschaftlich notwendige Arbeit zu begreifen und deren institutionelle Absicherung politisch einzufordern. Auf Seiten der bundesdeutschen Bildungspolitik blieb man zunächst bestrebt, das Problem zu bagatellisieren, bzw. es so weit wie möglich zu tabuisieren. Unzureichend schriftsprachlich gebildete Menschen passten nicht zum Selbstverständnis einer modernen Gesellschaft. Erst als der Druck anwaltschaftlicher Vertreter der funktionalen Analphabeten beständig größer wird, reagieren auch vereinzelt staatliche Stellen. In der Folgezeit setzt eine stärkere, wenngleich immer noch projektbezogene staatliche Unterstützung der deutschsprachigen Erwachsenenalphabetisierung ein. Der Beginn der staatlichen Projektförderung markiert einen durch eine neue Soziale Bewegung erzielten wichtigen Teilerfolg zur Verbesserung vorhandener struktureller Bildungsdefizite. Im Mittelpunkt der Erwachsenenalphabetisierung steht aber weiterhin basisnahes, innovatives, größtenteils ehrenamtliches Engagement. Es zeigt sich seit den 1980er Jahren in einer steigenden Zahl von lokalen und regionalen Initiativen, Bildungswerkstätten und Netzwerkbildungen. Exemplarisch sei die 1984 gegründete „Schreibwerkstatt für neue Leser und Schreiber e.V.“ genannt, aus der 1997 der „Bundesverband Alphabetisierung und Grundbildung e.V.“ entsteht. Ein Schwerpunkt der Interessenvertretung für die neu entdeckte Zielgruppe der funktionalen Analphabeten liegt in der Sensibilisierung der Öffentlichkeit für die spezifische Problemlage der Betroffenen. Bildungspolitisch kämpfte die Bewegung für die Implementierung und finanzielle Förderung von Grundbildung in der öffentlichen Weiterbildung. Auch wenn die Geschichte der Alphabetisierung in Deutschland einem Formwandel in Richtung Institutionalisierung und der damit einhergehenden Stabilisierung und Professionalisierung unterliegt, muss festgehalten werden, dass sie noch weit davon entfernt ist, ihren Bewegungscharakter abzustreifen. Eher bleibt mit Blick auf die aktuelle Situation zu festzustellen, dass die Alphabetisierungsbewegung mittlerweile zwar eindeutig organisierte Strukturen auf verschiedenen Ebenen umfasst, sie aber nicht vollständig in einer formalen Organisation aufgeht, sondern weiterhin als netzwerkförmiger Verbund von Personen, Gruppen und Organisationen existiert.Das stetige Anwachsen eines Bildungsproletariats in der Wissensgesellschaft des 21. Jahrhunderts ist ein prekäres soziales Thema, das von der parlamentarischen Demokratie und ihren Institutionen lange vernachlässigt bzw. unterschätzt wurde. Immer deutlicher zeigt sich, dass mit der beschleunigten ökonomischen Rationalisierung Millionen von Menschen die Chance auf Partizipation am öffentlichen Leben verwehrt ist. Sie erleiden den Ausschluss aus sozialen, politischen oder kulturellen Kontexten. Erst durch bürgerschaftliches Engagement und der daraus entstandenen Alphabetisierungsbewegung gelang es, in ersten Ansätzen eine grundlegende Bearbeitung des Themas Analphabetismus und Grundbildung in hoch entwickelten Industriestaaten auf den Weg zu bringen. Jens Korfkamp 